\title{
LETTER
}

\section{Ritonavir Polymorphism: Analytical Chemistry Approach to Problem Solving in the Pharmaceutical Industry}

\author{
Renan M. B. Dezena (iD \\ Preformulation Specialist \\ Pharmaceutical Industry, Campinas, SP, Brazil
}

Although multinational/innovative companies invest resources and time in studying the impact of different polymorphic forms on medicines, in Brazil the polymorphism issue is very recent. Control of the crystalline form or structure of solids should be carefully evaluated in the pharmaceutical industry because, despite the same chemical composition, polymorphs have distinct physicochemical properties, such as solubility, dissolution, chemical stability, appearance of the raw material, melting point and bioavailability. In addition, polymorphs can undergo phase conversion during a sequence of manufacturing steps, such as milling, micronization, drying, wet granulation, spray-drying and compaction.

The most widely known and well-known example in the history of the pharmaceutical industry regarding the influence of polymorphism leading to deviations in quality control and problems with the pharmacological effect in patients is the case of ritonavir.

The ritonavir molecule was developed by Abbott Laboratories in 1992. A new drug application was submitted in December 1995, marketing began in January 1996 and FDA approval was granted in March 1996. Ritonavir, sold under the tradename Novir ${ }^{\circledR}$, was introduced to the market as a gelatin capsule and as a liquid formulation [1]. Two years after its launch (early 1998), some batches began to fail the dissolution specifications and there were reports of clinical ineffectiveness, causing a market crisis for Abbott Laboratories [1]. Further investigation revealed the existence of a previously unknown new crystalline form that was thermodynamically more stable and much less soluble. This new polymorph was referred to as ritonavir Form II, with the originally known crystalline form being called ritonavir Form I [1]. The contents of the capsules were examined by microscopy. Crystals were detected on capsule filling that had a distinctly different crystal habit from bulk ritonavir [1]. The Form I morphology is generally observed as lath-shaped crystals or rods, whereas Form II crystals appear as thin needles (see Figure 1) [1].

The structures of the polymorphs were determined from x-ray powder diffraction (XRPD) and are shown in Figures 2 and 3. By analysing the diffractograms of the two polymorphic forms it was possible to verify that they present different XRPD profiles from each other: Form I has a characteristic peak at $6.75(2 \theta)$ whereas Form II has characteristic peaks at $9.51(2 \theta), 9.88(2 \theta)$ and $22.2(2 \theta)$ [1]. The crystal structures of Forms I and II were obtained from the Cambridge Crystallographic Data Center (CCDC), deposition numbers 710528 and 710529 , respectively, using the Mercury 4.3 program.

\section{Hydrogen bond evaluation}

In both polymorphic forms the total number of hydrogen bonds is the same, however the crystallographic network of hydrogen bonds is different [4].

Cite: Dezena, R. M. B. Ritonavir Polymorphism: Analytical Chemistry Approach to Problem Solving in the Pharmaceutical Industry. Braz. J. Anal. Chem., 2020, 7 (26), pp 12-17. doi: http://dx.doi.org/10.30744/brjac.2179-3425.letter.rmbdezena.N26 


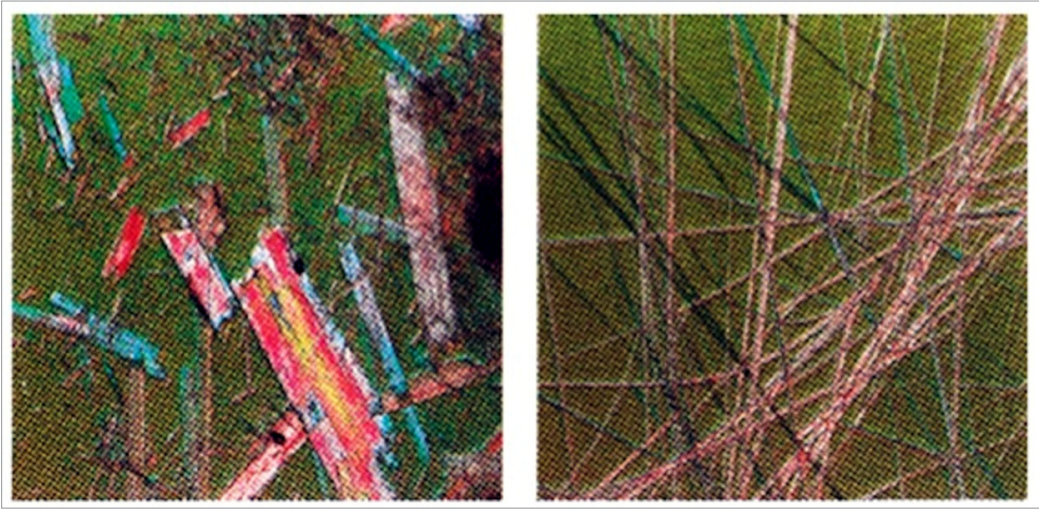

Figure 1. Micrograph of ritonavir crystals: (left) Form I; (right) Form II [1].

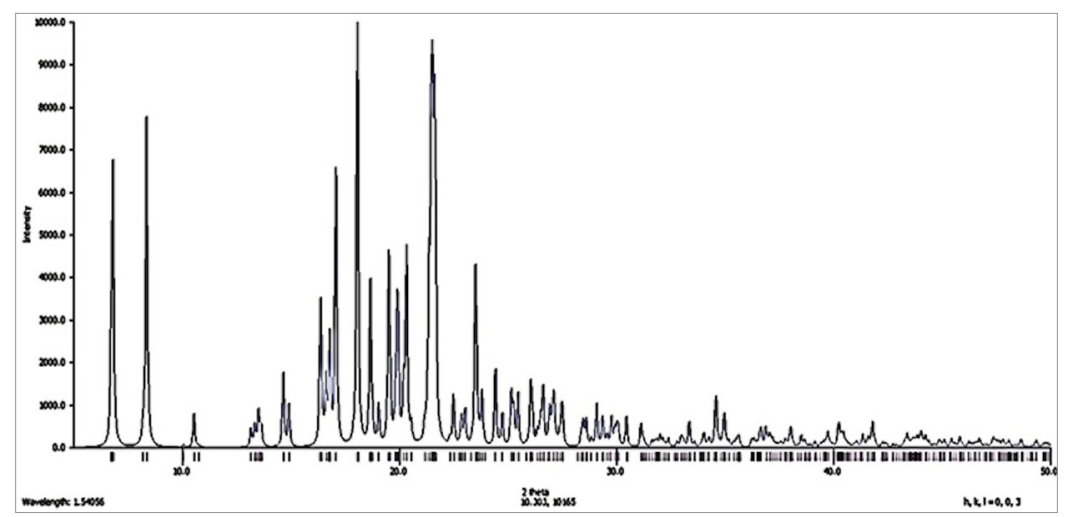

Figure 2. X-ray powder diffractogram of ritanovir Form I [2].

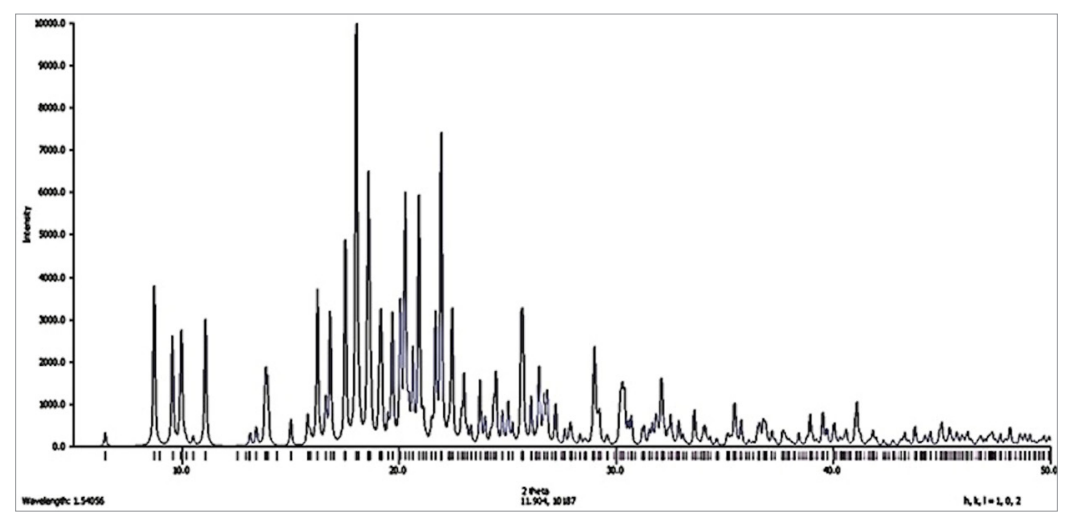

Figure 3. X-ray powder diffractogram of ritanovir Form II [3].

\section{Form I}

Figures 4 and 5 show the hydrogen bond arrangements for Form I: a continuous beta-like stack parallel to the short crystallographic axis due to alignment between the amide bonds of one molecule with the same functional group in the next molecule [4]; and pairing of these stacks due to hydrogen bonds between the alcohol of one molecule and the thiazole ring of a second related molecule [4]. 


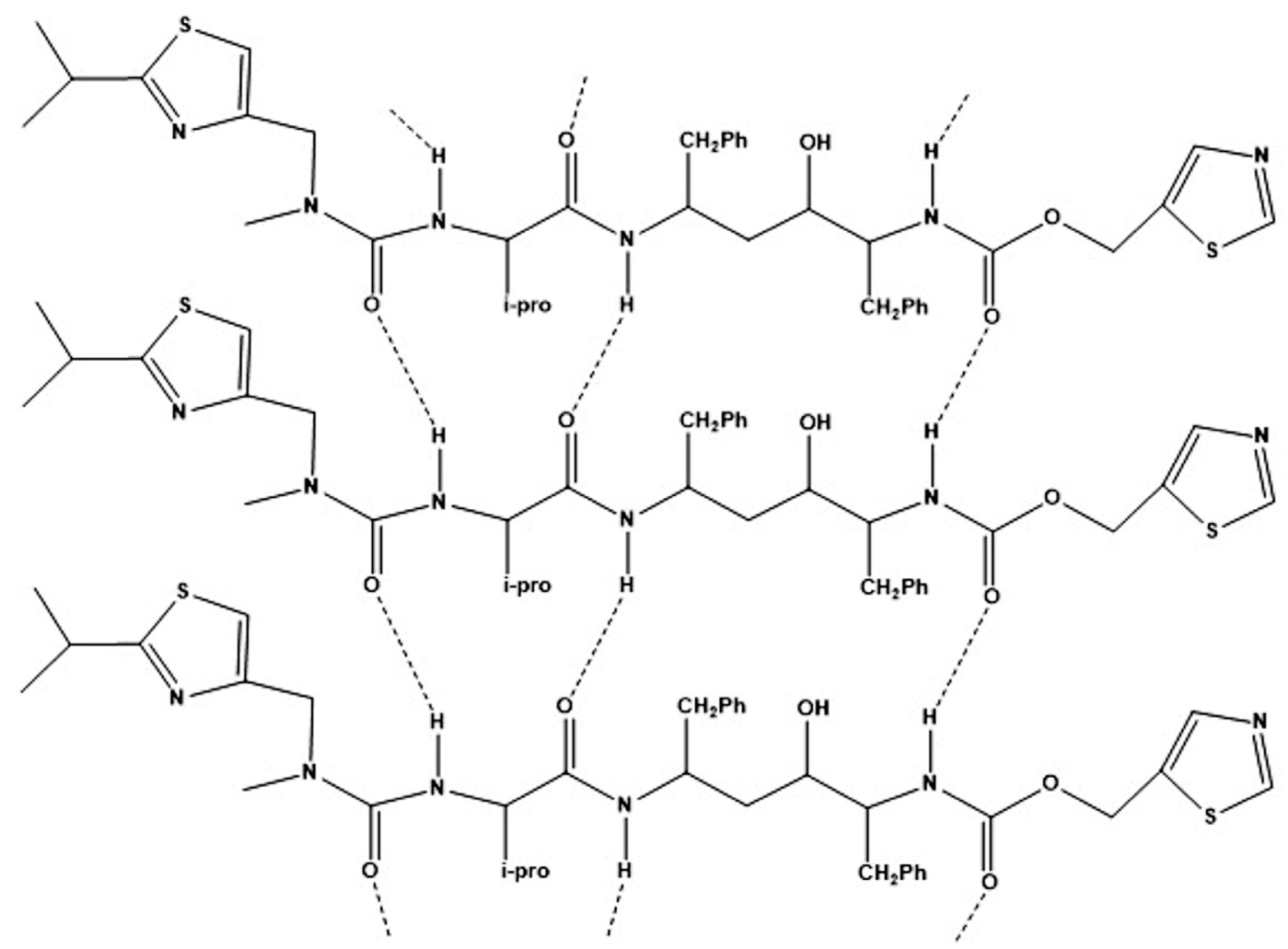

Figure 4. Crystallographic network for ritonavir Form I: beta-like stack [4].

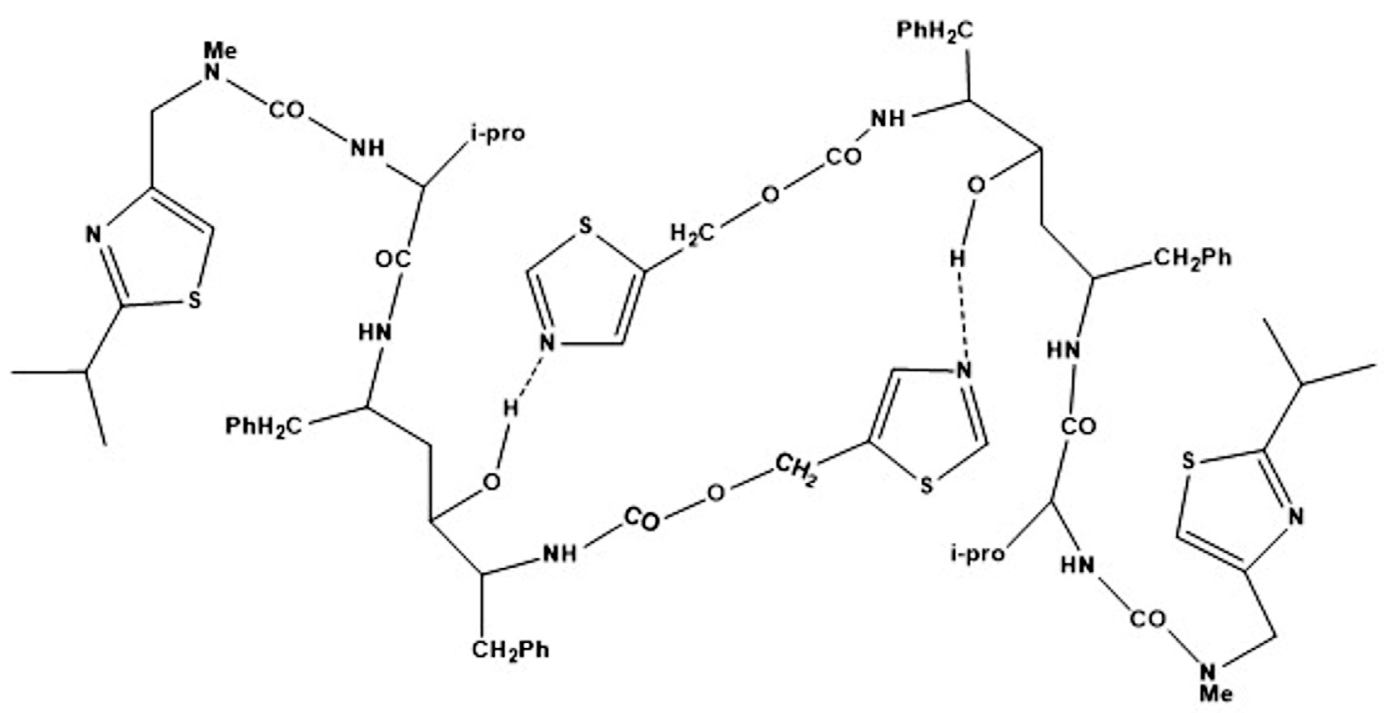

Figure 5. Crystallographic network for ritonavir Form I: twofold screw axis [4].

\section{Form II}

Figure 6 shows the hydrogen bond arrangement for Form II, from which it is possible to verify that the hydrogen bonds are strong and that both the hydrogen bond donors and acceptors are fully bonded [4]. 


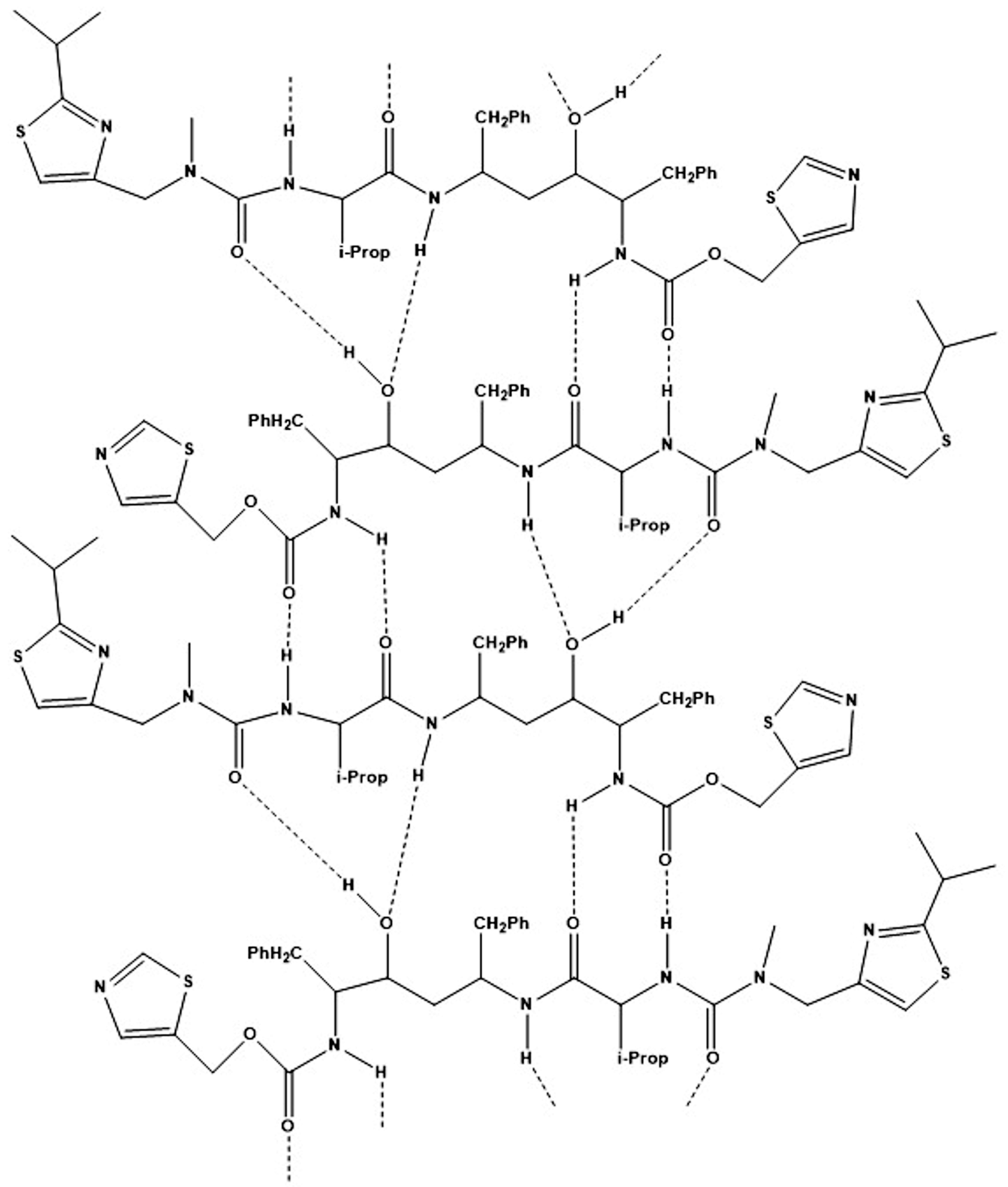

Figure 6. Crystallographic network for ritonavir Form II: needle growth occurs due to orientation promoted through hydrogen bond alignment [4].

Further, crystallographic elucidation of the polymorphic forms identifies the three torsion angles $(A, B$, C) represented in Figure 7, showing areas with significant differences between the different polymorphs [4]. 


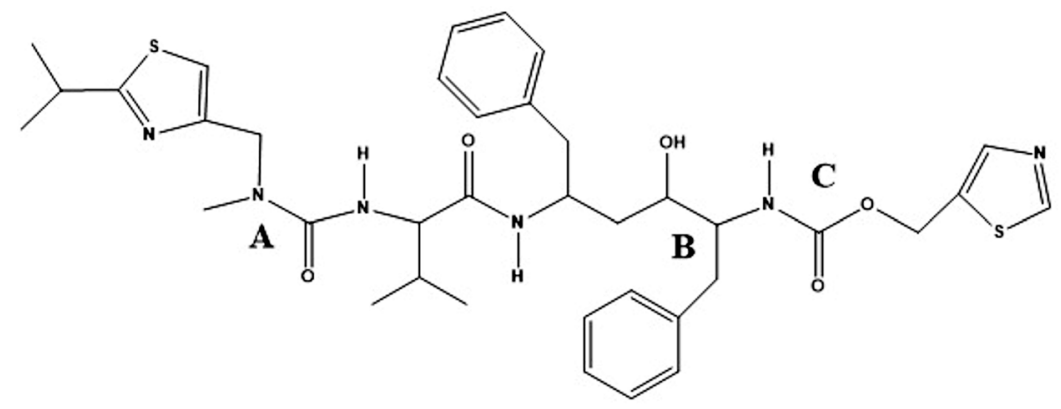

Figure 7. Three torsion angles (A, B, C) identified in ritonavir polymorphism [4].

The main question that still remained was how Form II emerged initially, given its resistance to nucleation [4]. Crystallization of Form II in supersaturated solutions can only occur if Form II seeds are present, so it has been hypothesized that a possible source for the formation of these original seeds would involve heterogeneous nucleation of an impurity of similar chemical structure capable of acting as a mold for Form II. Possible degradation steps for ritonavir are shown in Scheme 1 [4].
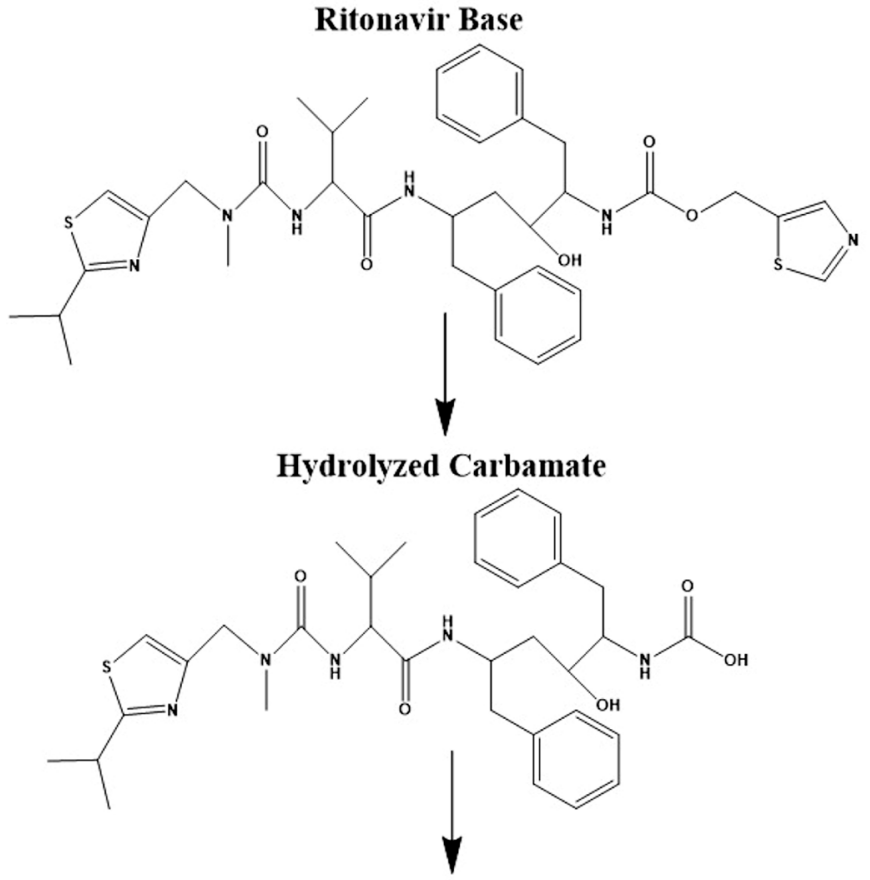

Cyclic Carbamate

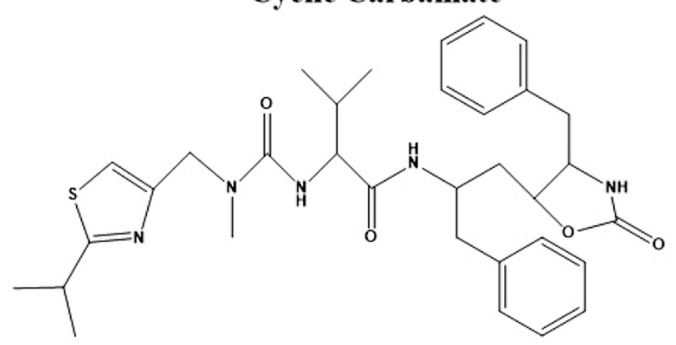

Scheme 1. Possible degradation steps for ritonavir.
Cyclic carbamate bonding of ritonavir yields a cis-like conformation ring and may serve as a template or seed for promoting the nucleation of Form II [4]. Using ritonavir characterization studies, the existence of conformational polymorphism ("cis" and "trans") around the carbamate bond was confirmed, leading to two unique crystallographic networks with significantly different solubility properties [4].

Nucleation of Form II requires the generation of a less stable conformation in solution, with subsequent organization for crystal growth [4]. Owing to the very low probability of nucleation, this new polymorphic form could remain hidden from scientific knowledge if it were not for the combination of a solution that was highly supersaturated (around $400 \%$ ) with respect to Form II and an unknown nucleation enhancer that was able to seed the solution heterogeneously [4].

\section{Summary}

This case study has demonstrated the need to invest financial resources in analytical chemistry to conduct research focused on identifying all possible polymorphs and determining the effects related to bioavailability and stability in pharmaceutical products. 


\section{Background}

Dealing with polymorphs seems imperceptible and mysterious but the initial investment in preformulation studies regarding identification, characterization and quality control of active pharmaceutical ingredient (API) polymorphism provides strategic insights to ensure drug quality and patent issues.

In this extremely competitive market between generic and innovative products, the API cannot be considered based solely from a molecular point of view; changes in solid-state properties also must be considered to ensure the interchangeability, efficacy and safety between these products.

Analytical chemistry has played a critical role in understanding the characterization and identification of different polymorphic forms through the use of analytical techniques such as microscopy, mid-infrared spectroscopy, differential scanning calorimetry, thermal gravimetric analysis, x-ray powder diffraction and nuclear magnetic resonance, thus becoming a well-established and well-known tool with essential practical application for the pharmaceutical industry [5-9].

\section{References}

1. Chemburkar, S. R.; Bauer, J.; Deming, K.; Spiwek, H.; Patel, K.; Morris, J.; Henry, R.; Spanton, S.; Dziki, W.; Porter, W.; et al. Org. Process Res. Dev., 2000, 4 (5), pp 413-417 (https://doi.org/10.1021/ op000023y).

2. Bauer, J.; Spanton, S.; Henry, R.; Quick, J.; Dziki, W.; Porter, W.; Morris, J. CCDC 710528: Experimental Crystal Structure Determination, 2007, (https://doi.org/10.5517/ccrvc86).

3. Bauer, J.; Spanton, S.; Henry, R.; Quick, J.; Dziki, W.; Porter, W.; Morris, J. CCDC 710529: Experimental Crystal Structure Determination, 2008, (https://doi.org/10.5517/ccrvc97).

4. Bauer, J.; Spanton, S.; Henry, R.; Quick, J.; Dziki, W.; Porter, W.; Morris, J. Pharm. Res., 2001, 18 (6), pp 859-866 (https://doi.org/10.1023/A:1011052932607).

5. Dezena, R. M. B. Br. J. Anal. Chem., 2017, 4 (16), pp 8-9.

6. Dezena, R. M. B.; Malta Jr., J. D. S. Br. J. Anal. Chem., 2017, 4 (15), pp 19-26.

7. Dezena, R. M. B.; Malta Jr., J. D. S.; Meurer, E. C.; Eberlin, M. N. Br. J. Anal. Chem., 2017, 4 (15), pp 27-34.

8. Senzel, A. J. Anal. Chem., 1973, 45 (8), pp 737A-737A (https://doi.org/10.1021/ac60330a742).

9. Yu, L. X.; Lionberger, R. A.; Raw, A. S.; Costa, R. D.; Wu, H.; Hussain, A. S. Adv. Drug Delivery Rev., 2004, 56 (3), pp 349-369 (https://doi.org/10.1016/j.addr.2003.10.012).

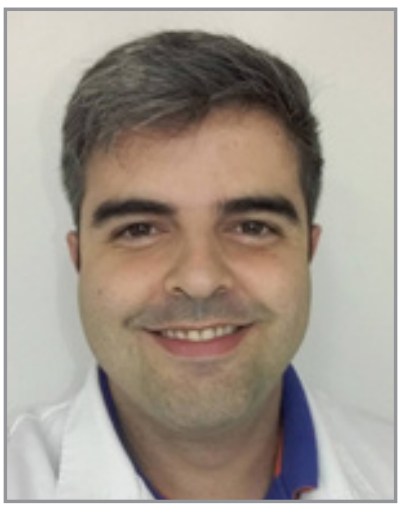

Renan M. B. Dezena is a preformulation specialist with graduation in pharmaceutical sciences from Pontifical Catholic University of Campinas, Brazil (2010), and specialization in Management of Research and Development of Drugs through the Institute of Sciences, Technology and Quality, Brazil (2014). He has experience in the pharmaceutical industry in the area of production, quality control and preformulation. Perform preformulation studies related to the development of pharmaceutical products through the following analytical techniques: Mass spectrometry (LC-MS/MS), Infrared Spectroscopy (MIR and NIR), Ultraviolet Spectroscopy, Liquid Chromatography (HPLC and UPLC), Gas Chromatography (GC-MS), Differential Scanning Calorimetry (DSC), Thermogravimetric Analysis (TGA), X-Ray Powder Diffraction (XRPD), Optical Microscopy, Particle Size Distribution by Laser Diffraction and Confocal Raman Microscopy.

CV: http://lattes.cnpq.br/1582491248654401 